\title{
Recent Progress in Sources, Biological Activity and Application of Astaxanthin
}

\author{
Xin-Yuan Cong ${ }^{1}$, Hui-Zhen Zhang ${ }^{1}$
}

\author{
${ }^{1}$ Qingdao University,School of Public Health Qingdao, Qingdao 266071, China
}

\begin{abstract}
Astaxanthin is a powerful biological antioxidant that exists in many living organisms. Various biological activities of astaxanthin confirmed in experimental animals studies. Astaxanthin has enormous potential and promising applications in food and medicine industry. In this paper, the sources,biological function,application of astaxanthin were reviewed.The current review has helpful for the further study on astaxanthin and its applications.
\end{abstract}

Keywords: Astaxanthin, Source, Physiological Activity, Application

\section{Introduction}

Astaxanthin $\left(\mathrm{C}_{40} \mathrm{H}_{52} \mathrm{O}_{4}\right)$ is a strong antioxidant and belongs to oxygenated carotenoids. It contains 11 unsaturated double bonds which making their chemical properties very unstable and easily damaged by external conditions such as light and heat ( $\mathrm{Li}$ et al., 2016). This structure provides electrons to reacts with free radicals, converting them into more stable products and terminating free radical chain reactions in a variety of organisms. Astaxanthin has many isomers, all-trans astaxanthin, 13-cis astaxanthin and 9-cis astaxanthin is the most commen structure. Astaxanthin has two chiral centers at the two-bonded ring structure of the conjugated double bond chain, which can produce three optical isomers: $3 \mathrm{~S}, 3^{\prime} \mathrm{S}, 3 \mathrm{~S}, 3^{\prime} \mathrm{R}$ and $3 \mathrm{R}, 3^{\prime} \mathrm{R}$ (MAOKA et al., 1985). Astaxanthin has extremely strong biological activity and important physiological function, which endows astaxanthin with extremely high application value. In this paper, the source, physiological activity and application of astaxanthins were summarized, and the related data were analyzed. To provide effective reference for the development of astaxanthin resources.

\section{Source of astaxanthin}

2.1 Chemical synthesis

As the various functions of astaxanthin are valued, the demand is also increasing. As the production of natural astaxanthin could't meet the demand, synthetic astaxanthin has also become an important source of astaxanthin. The synthetic astaxanthin is difficult process, and most of it is cis structure. Swiss Hoffmann-LaRoche successfully synthesized astaxanthin at the end of the twentieth century, with a content of about 5-10\% (Ambati et al., 2014). Synthetic astaxanthin is mainly used for animal feed because of its poor coloring ability and biological activity compared to natural astaxanthin(Higuera-Ciapara et al., 2006).

\subsection{Biogenetic derivation}

Astaxanthin in nature was ubiquitous in marine organisms such as fish, shrimp, shellfish and algae. And it was present in flamingo feathers and some fungi, such as phaffia rhodozyma or Rhodotorula rubra (Helliwell, 2010). The main natural sources of astaxanthin are Haematococcus pluvialis, Phaffia rhodozyma and Antarctic krill. Crustaceans are rich in astaxanthin, and the extraction of astaxanthin from discarded crustaceans was also an important resource (Chen et al., 1983).

\section{The function of astaxanthin}

3.1 Antioxidant function

Oxidative damage was caused by free radicals and reactive oxygen (ROS). Excessive oxidized molecules may react with proteins, lipids, and DNA through a chain reaction, resulting in oxidation of proteins and lipids and DNA damage, causing various diseases. The oxidized molecules could be inhibited by exogenous antioxidants such as astaxanthin. Astaxanthin terminates the oxidation reaction by quenching singlet oxygen and scavenging free radicals. It has been found that oxidative stress accelerates skin aging, and continuous supplementation of astaxanthin in the diet produced powerful antioxidant effects and alleviate skin aging, especially in obese people (Chalyk et al., 2017).

\subsection{Prevention of cardiovascular disease}

The anti-oxidation of astaxanthin may cure cardiovascular disease by improving oxidative stress. Atherosclerosis is an inflammatory disease of the arterial wall. Part of its pathological mechanism was caused by oxidative stress and inflammation. The severe clinical manifestations of myocardial infarction and stroke are mainly rupture or erosion of atherosclerotic plaque. Effective control and regulation

This article is published under the terms of the Creative Commons Attribution License 4.0

Author(s) retain the copyright of this article. Publication rights with Alkhaer Publications.

Published at: http://www.ijsciences.com/pub/issue/2019-03/

DOI: 10.18483/ijSci.2011; Online ISSN: 2305-3925; Print ISSN: 2410-4477 
of this response effectively alleviated the development of the disease and related complications (Pashkow et al., 2008). It has been found that astaxanthin could prevent cardiovascular diseases by improving lipid metabolism and inhibiting apoptosis (Chen et al., 2018). Astaxanthin shows a strong ability to reduce lipid oxidation, which helps to inhibit the formation of thrombosis and atherosclerotic plaque (Zuluaga et al., 2018). In a subchronic study of a 7-day oral supplementation with synthesis of astaxanthin derivatives in rat, the addition of astaxanthin reduced the level of plasma peroxidation products. It could reduce the damage of cardiomyocytes, inhibit the apoptosis of cardiomyocytes and improve the survival rate of cardiomyocytes. In addition, it reduced the myocardial infarct size by decreasing blood lipid levels, which had potential protective effect on the heart (Gross et al., 2006).

\subsection{Anti-aging effect}

Aging is the gradual loss of tissue or cellular function with age. Various theories such as telomere theory, free radical theory, mitochondrial theory and immunological theory have been proposed to explain the process of aging (Tosato et al., 2007). Free radical theory, as a possible explanation for the aging process, is accepted by more and more geriatricians. Oxidative stress caused by mitochondrial damage, plays an important role in accelerating aging. Budding yeast is an effective model for studying oxidative stress, programmed cell death and aging. Astaxanthin treatment has decreased ROS levels and lipid peroxidation, and increased superoxide dismutase activity in budding yeast. It showed that indicating that astaxanthin protects cells from oxidative stress-induced cell death. Astaxanthin could prolong the lifespan of antioxidant-deficient strains by scavenging ROS species ( $\mathrm{Sj}$ et al., 2019).

\subsection{Anti-inflammatory effect}

The antioxidant properties of astaxanthin also exhibit an anti-inflammatory effect. Protein tyrosine phosphorylation is an important mechanism that plays an important role in cell signal transduction, physiological functions and pathological processes (Lee et al., 2011). SHP-1 is a protein tyrosine phosphatase (PTP), a negative regulator of immune cytokine signaling, and SHP-1 deficiency had been found to promote the expression of inflammatory genes. When studying U937 monocytes, it was resported that astaxanthin was most likely to restore the level of SHP-1 and inhibit the production of inflammatory cytokines (Speranza et al., 2012). In studies of the effects of lipopolysaccharide-induced inflammatory responses, treatment with astaxanthin reduced the number of inflammatory cells. In particular, $100 \mathrm{mg} / \mathrm{kg}$ astaxanthin showed the same intensity of anti-inflammatory effects as prednisolone at a dose of $10 \mathrm{mg} / \mathrm{kg}$ (Ohgami et al., 2003).

\subsection{Hypoglycemic effect}

Diabetes is a metabolic disorder characterized by hyperglycemia and urinary glucose. Diabetes rely on hypoglycemic drugs to control blood sugar, but long-term use of hypoglycemic drugs lead to dependence and adverse reactions (El-Demerdash et al., 2005). Chen Zhiqiang's research indicates that astaxanthin has hypoglycemic effects on alloxan-modeled and adrenaline- and glucose-induced diabetic mice (Chen et al., 2008). Complications of diabetes are also difficult to treat. Long-term hyperglycemia can cause damage to many organs such as blood vessels, liver, kidney and nerves. Hyperglycemia and oxidative stress caused by diabetes are closely related to the cognitive function of patients. Studies have shown that astaxanthin has an inhibitory effect on neuronal apoptosis, and could protect neurons in diabetic mice from inflammatory damage, thereby improving cognition (Zhou et al., 2015).

\section{Astaxanthin application}

4.1 Health product

By improving the mitochondrial redox state, astaxanthin can alleviate the body's stress state caused by high-intensity exercise. At the same time, it also has the ability to lower blood uric acid and clear lactic acid, and it is expected to become a health care product for athletes to relieve fatigue and enhance muscle endurance (Wang et al., 2017). The elderly is characterized by a gradual loss of exercise capacity, and muscular dystrophy and decreased endurance are important factors in this intolerance. Astaxanthin could improve the muscle strength of elderly. And it has been shown that the right amount of astaxanthin combined with functional training can significantly improve the muscle strength, endurance and flexibility of the elderly (Liu et al., 2018). The edible history of astaxanthin has been in existence for more than 20 years and there has been no safety problems.

\subsection{Food Additive}

Astaxanthin has the effect of delaying the oxidation of fat and has a protective effect on lipids including fatty acids and cholesterol. It was discovered through research on the storage of raw mutton and cooked mutton products. Meat products containing 20-80 $\mathrm{mg} / \mathrm{kg}$ of natural astaxanthin could be placed in aerobic refrigeration for a longer time, helping to increase the shelf life of the product (Carballo et al., 2018). Research has so far reported no significant side effects of astaxanthin consumption in humans.

\subsection{Feed additives}

Astaxanthin is synthesized by plants and could be absorbed in animals without conversion. Animals taked in astaxanthin from the diet and stored it in tissues such as muscles and skin. In general, consumption of astaxanthin could blush the skin in animals. The addition of astaxanthin in diet maked the muscles appear bright orange-red, such as salmon and rainbow trout. A large amount of astaxanthin not only improved 
the appearance of fish but also increased the disease resistance of fish (Xun et al., 2012). Astaxanthin decreased tumor incidences and has a superior preventive effect toward photo-oxidative. Astaxanthin accumulation was observed when astaxanthin was fed to rats and, the main site of astaxanthin accumulation was the hairless skin of the tail (Rao et al., 2013). In rats cancer study, antioxidant enzymes such as superoxide dismutase and glutathione peroxidase levels significantly increased after diet with astaxanthin (Petri and Lundebye, 2007).

\subsection{Cosmetics}

Astaxanthin was widely used in the production of cosmetics due to its antioxidant and anti-aging functions, and it has been developed to anti-aging products with strong free radical scavenging activity (Jiang et al., 2017). Astaxanthin could absorb ultraviolet light with a wavelength between 240 and $400 \mathrm{~nm}$, and had a good sunscreen effect after compounding with titanium dioxide (Jiao et al., 2017). The mask made by combination of astaxanthin and triglyceride had better ability to scavenge free radicals than vitamin E, indicating a strong antioxidant capacity (Cheng et al., 2018). One study found that sunscreen and serum added astaxanthin which has great stability and applicability (Yang et al., 2015).

\section{Conclusion}

Astaxanthin has great demand in food, feed, nutraceutical and cosmetics applications. Meanwhile, astaxanthin has broad development potential and great development space in medicine fields. In conclusion, astaxanthin is suggested as a promising product in health promotion, futher study will find more physiological activity and application.

Conflicts of Interest: The authors declare no conflict of interest.

\section{References:}

1. Ambati, R., Phang, S., Ravi, S., Aswathanarayana, R., 2014 Astaxanthin: Sources, Extraction, Stability, Biological Activities and Its Commercial Applications-A Review. Marine Drugs 12 (1), 128-152.

2. Carballo, D.E., Caro, I., Andrés, S., Giráldez, F.J., Mateo, J., 2018. Assessment of the antioxidant effect of astaxanthin in fresh, frozen and cooked lamb patties. Food Research International 111, 342-350.

3. Chalyk, N.E., Klochkov, V.A., Bandaletova, T.Y., Kyle, N.H., Petyaev, I.M., 2017. Continuous astaxanthin intake reduces oxidative stress and reverses age-related morphological changes of residual skin surface components in middle-aged volunteers. 48, 40-48.

4. Chen, H., Meyers, S.P., 1983. Ensilage Treatment of Crawfish Waste for Improvement of Astaxanthin Pigment Extraction. Journal of Food Science 48 (5), 1516-1520.

5. Chen, Z., Ren, L., Jiang, Z., Yang, H., 2008. Study on the hypoglycemic effect of astaxanthin. Food Science (07), 402-404.

6. Cheng, X., Xiong, Y., Yang, M., Zhu, M., 2018. Preparation of astaxanthin mask from Phaffia rhodozyma and its evaluation. Process Biochemistry.

7. El-Demerdash, F.M., Yousef, M.I., El-Naga, N.I.A., 2005. Biochemical study on the hypoglycemic effects of onion and garlic in alloxan-induced diabetic rats. Food and Chemical
Toxicology 43 (1), 57-63.

8. Gross, G.J., Hazen, S.L., Lockwood, S.F., 2006. Seven day oral supplementation with Cardax TM (disodium disuccinate astaxanthin) provides significant cardioprotection and reduces oxidative stress in rats. Molecular and Cellular Biochemistry 283 (1-2), 23-30.

9. Helliwell, J.R., 2010. The structural chemistry and structural biology of colouration in marine crustacea. Crystallography reviews 16 (3), 231-242.

10. Higuera-Ciapara, I., Félix-Valenzuela, L., Goycoolea, F.M., 2006. Astaxanthin: A Review of its Chemistry and Applications. Critical Reviews in Food Science and Nutrition 46 (2

11. ), 185-196

12. Jiang, G., Zhou, L., Wang, Y., Zhu, M., 2017. Astaxanthin from Jerusalem artichoke: Production by fed-batch fermentation using Phaffia rhodozyma and application in cosmetics. Process Biochemistry 63, 16-25.

13. Jiao, X., Chi, L., Chen, Z., 2017. Study on the sunscreen effect of natural astaxanthin. Guangdong Chemical Industry (18), 92-93.

14. Lee, S.M., Kim, E.J., Suk, K., Lee, W.H., 2011. Synthetic peptides containing ITIM-like sequences of IREM-1 inhibit BAFF-mediated regulation of interleukin- 8 expression and phagocytosis through SHP-1 and/or PI3K. Immunology 134 (2), 224-233

15. Li, Y., Liu, T., Tang, S., 2016. Advances in the stability and biological activities of natural astaxanthin. Journal of guangdong pharmaceutical university (01), 126-130.

16. Liu, S.Z., Ali, A.S., Campbell, M.D., Kilroy, K., Shankland, E.G., Roshanravan, B., Marcinek, D.J., Conley, K.E., 2018. Building strength, endurance, and mobility using an astaxanthin formulation with functional training in elderly. Journal of Cachexia, Sarcopenia and Muscle 9 (5), 826-833.

17. Maoka, T., Masaaki, K., Nobuyuki, K., Matsuno, T., 1985. Stereochemical Investigation of Carotenoids in the Antarctic Krill Euphausia superba. Bulletin of the Japanese Society of Scientific Fisheries (51(10)), 1671-1673.

18. Ohgami, K., Shiratori, K., Kotake, S., Nishida, T., Mizuki, N., Yazawa, K., Ohno, S., 2003. Effects of astaxanthin on lipopolysaccharide-induced inflammation in vitro and in vivo. Investigative ophthalmology \& visual science 44 (6), 2694.

19. Pashkow, F.J., Watumull, D.G., Campbell, C.L., 2008 Astaxanthin: A Novel Potential Treatment for Oxidative Stress and Inflammation in Cardiovascular Disease. The American Journal of Cardiology 101 (10), S58-S68.

20. Petri, D., Lundebye, A., 2007. Tissue distribution of astaxanthin in rats following exposure to graded levels in the feed. Comparative Biochemistry and Physiology Part C: Toxicology \& Pharmacology 145 (2), 202-209.

21. Rao, A.R., Sindhuja, H.N., Dharmesh, S.M., Sankar, K.U., Sarada, R., Ravishankar, G.A., 2013. Effective Inhibition of Skin Cancer, Tyrosinase, and Antioxidative Properties by Astaxanthin and Astaxanthin Esters from the Green Alga Haematococcus pluvialis. Journal of Agricultural and Food Chemistry 61 (16), 3842-3851.

22. Shen, C., Wang, L., Wang, X., 2018. Advances in the study of astaxanthin in atherosclerotic cardiovascular disease. Medical Recapitulate 24 (03), 417-421.

23. Sj, S., Veerabhadrappa, B., Subramaniyan, S., Dyavaiah, M., 2019. Astaxanthin enhances the longevity of Saccharomyces cerevisiae by decreasing oxidative stress and apoptosis. FEMS Yeast Res 19 (1).

24. Speranza, L., Pesce, M., Patruno, A., Franceschelli, S., Lutiis, M.A.D., Grilli, A., Felaco, M., 2012. Astaxanthin Treatment Reduced Oxidative Induced Pro-Inflammatory Cytokines Secretion in U937: SHP-1 as a Novel Biological Target. Marine Drugs 10 (12), 890-899.

25. Tosato, M., Zamboni, V., Ferrini, A., Cesari, M., 2007. The aging process and potential interventions to extend life expectancy. Clin Interv Aging 2 (3), 401-412.

26. Wang, J., Ruan, C., Wu, L.,2017. Effects of astaxanthin supplementation on motor function. Sports science and technology literature bulletin (11), 168-169. 
27. Xun, H., Cao, B., Chen, J., Wei, G., 2012. Biological functions and applications of astaxanthin. Feed and Husbandry (8), 45-48.

28. Yang, T., Liao, Me., He Y., Liu. S., Li, P., 2015. Preliminary research on the sunscreen cream based on astaxanthin. Science of household chemicals 38 (04), 29-32.

29. Zhou, X., Zhang, F., Hu, X., Chen, J., Wen, X., Sun, Y., Liu, Y., Tang, R., Zheng, K., Song, Y., 2015. Inhibition of inflammation by astaxanthin alleviates cognition deficits in diabetic mice. Physiology \& Behavior 151, 412-420.

30. Zuluaga, M., Gueguen, V., Letourneur, D., Pavon-Djavid, G., 2018. Astaxanthin-antioxidant impact on excessive Reactive Oxygen Species generation induced by ischemia and reperfusion injury. Chemico-Biological Interactions 279, 145-158. 